\title{
Cognitive Radio: Spectrum Sensing Problems in Signal Processing
}

\author{
J. Christopher Clement \\ VIT University \\ Vellore \\ Tamil Nadu, India
}

\author{
Kishore V. Krishnan \\ VIT University \\ Vellore \\ Tamil Nadu, India
}

\author{
A. Bagubali \\ VIT University \\ Vellore \\ Tamil Nadu, India
}

\begin{abstract}
The electromagnetic radio spectrum is a natural resource. Effective Utilization of this natural resource is a Challenging task in Present Day Wireless Communication. Cognitive Radio is an emerging trend in wireless communication to Combat for spectral scarcity. Cognitive radio is an intelligent wireless communication system that is aware of its surrounding environment and uses the methodology of learning, and understanding from the environment and adapting to it. Advanced signal processing techniques are used in Cognitive Radio Network to achieve this intelligence. Spectrum Sensing is one of such a signal processing to identify spectrum holes which can be temporarily used without interfering Primary User. Every cognitive radio receiver is incorporated with Spectrum Sensing. There are various existing techniques to detect the spectrum of which some widely used are presented in this thesis.
\end{abstract}

\section{General Terms}

Your general terms must be any term which can be used for general classification of the submitted material such as Pattern Recognition, Security, Algorithms et. al.

\section{Keywords}

CR - Cognitive Radio, CSD - Cyclic Spectrum Density, PSD - Power Spectrum Density, RF Spectrum- Radio Frequency Spectrum.

\section{INTRODUCTION}

According to the report published by The Federal Communications Commission (FCC) in November 2002 [1].

1) Some frequency bands in the spectrum are largely unoccupied most of the time;

2) Some other frequency bands are only partially occupied;

3) The remaining frequency bands are heavily used.

In every wireless Communication there exists a primary user (Licensed User) who is authorized to access specific part of the spectrum. FCC has opened a way for Cognitive radio network to allocate this Primary users spectrum to secondary unlicensed user without intervening primary user communication [1]. The underutilization or the bands of frequencies which are not used by the primary user at particular time and at particular geographical location are called spectrum holes[2]. Spectrum utilization can be improved significantly by making it possible for a secondary user (who is not being serviced) to access a spectrum hole unoccupied by the primary user at the right location and the time in question [2]. This paper will try to address the task of identifying the spectrum holes in the local neighborhood of radio receiver. The conventional way of spectrum sensing is measuring energy of radio frequency spectrum over wide band of interest. In cognitive radio terminology spectrum sensing involves measuring energy in various dimensions like time, space, frequency and code [2]. It also tries to identify types of signal occupying the spectrum such as modulation, bandwidth, and carrier frequency etc.

Rest of the paper is organized as follows. Section II defines Spectrum holes as white, Gray, and Black Spaces. Section III describes spectrum sensing techniques, their advantages, and disadvantages. And finally section IV concludes the paper.

\section{SPECTRUM HOLES}

A licensed spectrum band that is not used at some geo graphic locations and at the time of question is called spectrum hole. Generally spectrum holes are broadly classified into temporal spectrum holes and spatial spectrum holes as shown in Fig respectively [3]. A temporal spectrum hole is unoccupied by the primary user during the time of sensing [3]. Hence, this band can be utilized by CR users in the current time slot. As it works in time domain, spectrum sensing of this kind does not demand complex signal processing [3]. A spatial spectrum hole is a frequency band which is free for secondary user at some spatial areas; and therefore can be utilized by CR users well outside this area. Spatial sensing of spectrum hole requires complex signal processing algorithms.

In terms of the amount of power a band possess, power spectra of incoming RF stimuli is classified into three types as [2]

1) Black spaces, which are occupied by high-power "local" interferers some of the time.

2) Grey spaces, which are partially occupied by low-power interferers.

3) White spaces, which are free of RF interferers except for white Gaussian noise.

Among this three, White spaces (for sure) and grey spaces (to a lesser extent) can be used by unlicensed operators if accurate sensing technique is adapted, and Black spaces cannot be used because usage of this space will cause interference to the primary user 


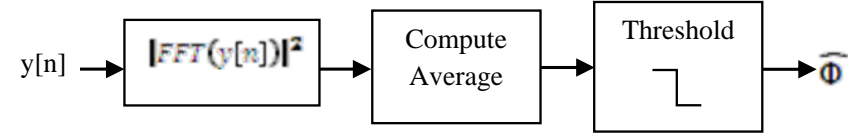

Fig.1 Energy Based Spectrum Sensing

\section{SPECTRUM SENSING TECHNIQUES}

\subsection{Energy Based Spectrum Sensing}

Energy based spectrum Detection is the simplest technique used for spectrum sensing [3]. Periodogram estimation is the other name of Energy based spectrum detection. Principle of energy detector is finding the energy of the received signal and compares that with the threshold [4]. Decision is made based on the comparison of signal to noise ratio of the received signal with that of threshold [5]. Advantages of energy based detectors are [5-7]

1) No prior knowledge of the primary users signal is required; hence the design is complex free.

2) Useful for wide band spectrum sensing. Sensing of number of sidebands can be realized by simply scanning the power spectral density.

Disadvantage of energy based detectors include, [5-7]

1) Selection of threshold

2) In case of low signal to noise ratio they are inefficient in discriminating interference from primary signal.

Idea of detection is explained with following equations and also shown in fig 1 . Received RF signal is written in the form as [5].

$$
y[n]=x[n]+w[n]
$$

where $x[n]$, is a primary users signal to be detected and $w[n]$ is additive white Gaussian noise with zero mean and unit variance.

Energy of a finite duration signal is usually given by

$$
E=\sum_{n=0}^{N}|x[n]|^{2}
$$

And the same is the metric used for comparison. The solution is all about discriminating between two hypotheses as

$$
\emptyset= \begin{cases}\emptyset_{0}, y[n]=x[n], & \text { if } E<\mu \\ \phi_{1}, y[n]=x[n]+w[n], & \text { if } E>\mu\end{cases}
$$

$\emptyset_{1}$ is the hypotheses for presence of primary signal, and $\emptyset_{0}$ indicates hypotheses for the absence of primary user signal. $\mu$ in (3) denotes threshold of comparison which could be found from distribution of test statistics.

In addition, test statistics of energy based detectors follow centralized chi square distribution for $\emptyset_{0}$ and non-centralized chi square distribution for $\emptyset_{1}$ respectively

\subsection{Waveform Based Sensing}

Waveform based sensing make use of the correlation property possessed by RF signal. In wireless communication, known patterns are transmitted with the original signal for some desired operations like channel estimation etc. [3]. These regular patterns are called as pilots. Pilots are attached either at the beginning or at the end of transmitted signal bursts. Pilots help receiver to perform time, frequency synchronization. Say these pilots are known at the receiving side, Correlation between received signal and the known pilots will give the metric for spectrum hole detection as described below.

If the same receiving model given in (1) is assumed, then metric of comparison using correlation is [8]

$$
E=\operatorname{Re}\left[\sum_{n=0}^{N-1} y[n] x^{*}[n]\right]
$$

where* denotes complex conjugate of the transmitted signal $x(n)$.

When the primary user signal occupies the band, (4) could be rewritten as

$$
E=\sum_{n=0}^{N-1}|x[n]|^{2}+R e\left[\sum_{n=0}^{N-1} w[n] x^{*}[n]\right]
$$

whereas when the band is free, metric is simply the correlation between noise and transmitted signal and is given below,

$$
E=\operatorname{Re}\left[\sum_{n=0}^{N-1} w[n] x^{*}[n]\right]
$$

The decision is made based on comparing the metric with fixed threshold value. Sensing is all about deciding between two hypotheses one for metric in (5) and the other for metric in (6). As like Energy based detector, test statistics follow central chi square distribution for hypotheses in (5) and noncentral chi square distribution for hypotheses in (6) respectively [3]. Waveform based sensing will perform well even under low signal to noise ratio unlike energy based detectors [3]. Disadvantage of waveform based sensing include prior knowledge of received signal is needed at the receiver [3]

\subsection{Cyclostationary Based Sensing}

Signals exhibit periodicities in its statistics are called Cyclostationary signals [9]. Cyclostationarity in signals may be caused by modulation and coding or even be intentionally produced to aid channel estimation and synchronization [10]. Such periodicity can be utilized for detection of a random signal with a particular modulation type in a background of noise and other modulated signals [3]. This is called cyclostationary detection. Cyclostationary detection is achieved from cyclic autocorrelation function of the received signal or, equivalently, its two-dimensional spectrum correlation function [11]. Due to its noise rejection property, cyclostationary detection works better even under very low SNR region [3], where the traditional signal detection method, such as the Energy Detection, fails.

Transmitted signals are Cyclostationarity, meaning they are spectrally correlated whereas noise signals are wide sense stationary in the sense they are uncorrelated [9]. Therefore cyclic spectral density is used for discriminating primary users from interferers. CSD is given as,

$$
\eta(f, \alpha)=\sum_{\tau=-\alpha}^{\alpha} C_{y}^{\alpha}(\tau) e^{-i 2 \pi f \tau}
$$

where

$$
C_{y}^{\alpha}(\tau)=E\left[y(n+\tau) y^{*}(n-\tau) e^{-i 2 \pi \alpha n}\right]
$$




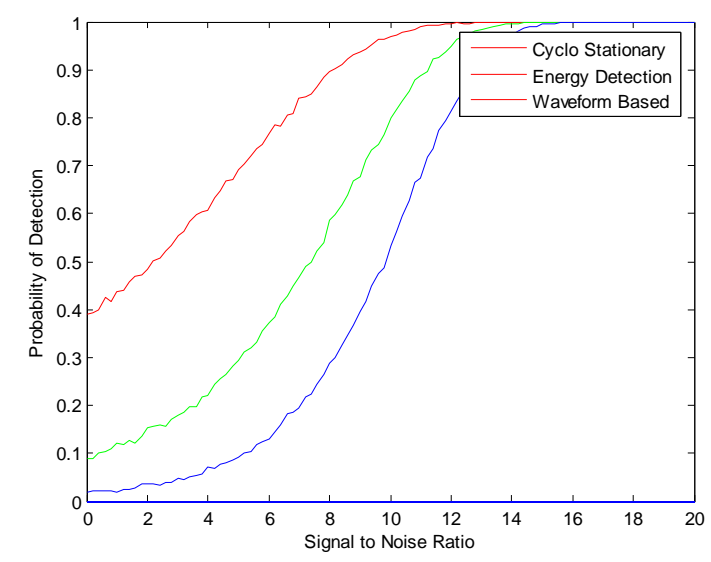

Fig.2 Sensing Performance

Is the cyclic auto correlation function [9] and $\alpha$ is the cyclic frequency which can be extracted from transmitted signal. Cyclic auto correlation will have nonzero values at integer multiples of fundamental frequency [9]. Hence CSD will have peaks when $\alpha$ is equal to fundamental frequency of transmitted signals [9]

\subsection{Multi Taper Spectral Estimation}

It is described in section energy detection method works on wide band of RF spectrum meaning that it looks on the entire sub bands simultaneously. As some sub-bands are occupied with transmit powers of licensed users and other bands are occupied with noise power alone, power spectral estimator is expected to have highest dynamic range [3]. To meet this requirement, the widest band is now broken down into small sub bands and same energy detection method is implemented. Filter banks of number $\mathrm{N}$ is used for this purpose to span the entire spectrum. If base band equivalent impulse response of the filter is $h[n]$, then

$$
h_{i}[n]=h[n] e^{-i 2 \pi f n}
$$

is the $\mathrm{i}^{\text {th }}$ band pass equivalent impulse response of filter $1 \leq i \leq N$ with center frequency $\mathrm{i} / \mathrm{N}[3] . h[n]$ is carefully chosen to reduce power leakage from sideband to the band of interest

For pages other than the first page, start at the top of the page, and continue in double-column format. The two columns on the last page should be as close to equal length as possible.

PSD metric from energy detector is modified here as follows,

$$
E=\sum_{n=0}^{N-1}\left|y[n] h[n] e^{-i 2 \pi f n}\right|^{2}
$$

Where $h[n]$ is same as in and it could be a simple rectangular window. As rectangular window does not have small side lobe leakage, it provides poor spectral dynamic range [3]. Multi tapering Spectral estimation is shown in fig 2. Most of the windows used in signal processing do not offer minimum variance of estimation during preprocessing of FFT stage [3]. Minimum variance of estimation could be obtained using special sequence called slepian sequences [2] which have their Fourier transform of maximal energy concentration in narrow main band and least in side band. In addition they are orthogonal to each other. Since they are orthogonal to each other, estimation results from various sub bands are uncorrelated and therefore estimation variance is reduced [2].
Disadvantages of multi tapering Spectral estimation include complexity in the design [2].

\subsection{Covariance Based Spectral Detection}

Signal received at the $\mathrm{CR}$ receiver exhibits correlation nature due to disperse in channel and multiple receive antenna. Correlation nature of received signal gives a way of using covariance matrix to detect the occupancy of primary signal.

Signal model of the received signal is

$$
y[n]=P x[n]+w[n]
$$

The value $P=1$ indicates occupancy of primary signal and $p=$ 0 indicates absence of the signal respectively. Covariance of the received signal is estimated using the estimator [3].

$$
\hat{C}=\frac{1}{N} \sum_{n=L-1}^{N-1}\left(y[n] y^{H}[n]\right)
$$

where $y[n]=[y[0] y[1] \cdots y[N-L-1]]$ column vector of size $L$. After simple mathematic manipulations we can get,

$$
\hat{C}=P C_{S}+\sigma^{2} I_{L}
$$

Where $C_{s}$ is the covariance matrix of signal column vector [3] of length $L$ as $x[n]=[x[0] x[1] \cdots x[N-L-1]], \sigma^{2}$ is the noise variance, and $I_{L}$ is Identity matrix of size $L \mathrm{X} L$. Let $\lambda_{\max }$, and $\lambda_{\min }$ be the maximum and minimum eigen values of covariance matrix respectively. The test metric for covariance based estimation is formulated as follows [3]

$$
E=\frac{\lambda_{\max }}{\lambda_{\min }}
$$

Decision is based on comparing the metric in (14) with the threshold $\mu$.

$$
\hat{\theta}=\left\{\begin{array}{l}
\theta_{1} \text { if } E>\mu \\
\theta_{1} \text { if } E<\mu
\end{array}\right.
$$

where $\theta_{0}$ and $\theta_{1}$ are the hypotheses for $\mathrm{P}=0$ and $\mathrm{P}=1$ respectively, and $\mu$ is the threshold of comparison

\subsection{Wavelet Based Sensing}

In wavelet based sensing, wide spectrum band is thought to be $\mathrm{k}$ number of disjoint sub bands. Power Spectral Characteristics are assumed to be smooth within a band, and the same is discontinuous across adjacent sub bands. Let the signal received from all primary users is represented as

$$
y[n]=\sum_{k=0}^{K} P_{k} x_{k}[n]+w[n]
$$

Where $P_{k}=1$ denotes presence of kth primary user signal $P_{k}=0$, denotes no signal from kth primary user respectively, and $w[n]$ is additive white Gaussian noise with unit variance and zero mean. As mentioned before, signal spectral density is flat during sub band of interest $\omega_{k} \leq$ $\omega \leq \omega_{k+1}$. In addition, noise spectral density is flat throughout all frequencies $\omega[3]$. Estimation of PSD of received signal is [12]

$$
\hat{S}_{y}(f)=\frac{1}{N} \sum_{k=0}^{\frac{N}{L}-1}\left|\sum_{l=0}^{L-1} y(l+k L) e^{-\frac{i 2 \pi f t}{N}}\right|^{2}
$$




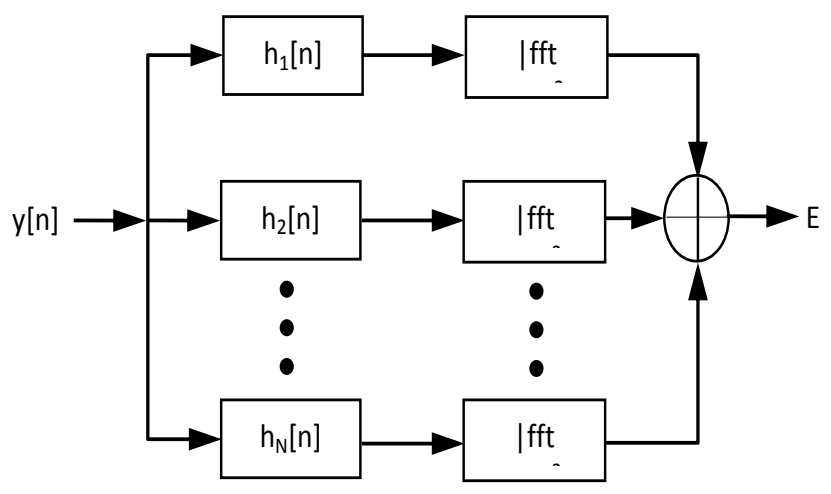

Fig.3 Multi Taper Spectrum Estimation

The task now is to identify the nonzero coefficients $\alpha_{k}$ which reflect pre occupancy of the spectrum by finding the frequency boundaries. Let $\psi(f)$ be the wavelet smoothing function used to extract features of $\hat{S}_{y}(f)$. Wavelet transform of estimated spectral density is given by the convolution operator. [3, 12]

$$
\widehat{W}_{y}(s ; f)=\hat{S}_{y}(f) * \psi_{s}(f)
$$

Where * denotes convolution, and $\psi_{s}(f)$ is the dilated version of $\psi(f)$ by a dyadic scale factor of $s=2^{j} ; 1 \leq j \leq J$ as [12]

$$
\psi_{s}(f)=\frac{1}{s} \psi\left(\frac{f}{s}\right)(19)
$$

Frequency boundaries are manipulated from the derivative of local maxima of wavelet modulus

$$
\hat{f}_{k}=\arg \frac{\max }{f_{\min } \leq f \leq f_{\max }}\left|\prod_{j=1}^{J} W_{y}^{\prime}\left(2^{j} ; f\right)\right|
$$

Coefficients of interest in terms of spectral boundaries are

$$
\hat{\alpha}_{k}=\frac{1}{\hat{f}_{k}-\hat{f}_{k-1}} \int_{\hat{f}_{k-1}}^{\hat{f}_{k}} \hat{S}_{y}(f) d f-N_{0}, 1 \leq k \leq K(21)
$$

When $\hat{\alpha}_{k} S$ are zero, Corresponding spectrum band is decided to be unoccupied [3].

\section{CONCLUSION}

Cognitive radio is one of the mile stone in wireless communications. Algorithms discussed in this thesis have their own advantages and disadvantages. Some tradeoff between complexity and performance is to be made to decide good algorithm for specific application. Some precise sensing algorithms like cooperative detection etc. are emerging day to day.

\section{REFERENCES}

[1] Federal and C. Commission, "Spectrum Policy Task Force," Rep. ET Docket no,02-135, Nov. 2002.

[2] S. Haykin, "Cognitive Radio: Brain-Empowered Wireless Communications," IEEE Journal On Selected Areas In Communications, vol. 23, NO. 2, February 2005.

[3] J. Ma, et al., "Signal Processing in Cognitive Radio," Proceedings of the IEEE, vol. 97, No. 5, pp. 805-822, May 2009.

[4] G. Ganesan and Y. Li, "Agility improvement through cooperative diversity in cognitive radio," in Proc IEEE Global Telecomm. Conf. (Globecom), St. Louis, Missouri, USA, vol. 5, pp. 2505-2509, Nov./Dec. 2005.

[5] F. Digham, et al., "On the energy detection of unknown signals over fading channels," in Proc. IEEE Int. Conf.Commun., vol. 5, Seattle, Washington, USA, pp. 3575-3579, May 2003.

[6] A. Ghasemi and E. Sousa, "Optimization of spectrum sensing for opportunistic spectrum access in cognitive radio networks," in Proc.IEEE Consumer Commun. and Networking Conf., Las Vegas, Nevada, USA, pp. 1022 1026, Jan. 2007.

[7] D. Cabric, et al., "Implementation issues in spectrum sensing for cognitive radios," in Proc. Asilomar Conf. on Signals, Systems and Computers, Pacific Grove, California, USA, vol. 1, pp. 772-776, Nov. 2004.

[8] H. Tang, "Some physical layer issues of wide-band cognitive radio systems," in Proc. IEEE Int. Symposium on New Frontiers in Dynamic Spectrum Access Networks, Baltimore, Maryland, USA, pp. 151-159, Nov. 2005.

[9] W. A. Gardner, "Exploitation of spectral redundancy in cyclostationary signals," IEEE SP Magazine, pp. 33-35, Apr 1991.

[10] N. Han, et al., "Spectral correlation based signal detection method for spectrum sensing in IEEE 802.22 WRAN systems," Proc. IEEE Int. Conf. Advanced Communication Technology, vol. 3, Feb. 2006.

[11] J. Lund'en, et al., "Spectrum sensing in cognitive radios based on multiple cyclic frequencies," in Proc. IEEE Int. Conf. Cognitive Radio Oriented Wireless Networks and Commun. (Crowncom), Orlando, Florida, USA, July/Aug. 2007.

[12] Z. Tian and G. B. Giannakis, "A Wavelet Approach to Wideband Spectrum Sensing for Cognitive Radios," IEEE SIgnal Processing Magazine, Nov 2006 